\title{
ON THE BOUNDARY POINT PRINCIPLE FOR ELLIPTIC EQUATIONS IN THE PLANE ${ }^{1}$
}

\author{
BY J. K. ODDSON
}

Communicated by J. B. Diaz, November 27, 1967

1. Let $D$ be an open connected subset of $E^{n}(n \geqq 2)$ and denote by $\mathfrak{L}_{\alpha}(D)$ the class of second order uniformly elliptic operators of the form $L=\sum_{i, j=1}^{n} a_{i j} \partial^{2} / \partial x_{i} \partial x_{j}$ with coefficients defined in $D$ and satisfying there the condition $\sum_{i, j=1}^{n} a_{i j} \xi_{i} \xi_{j} \geqq \alpha \sum_{i=1}^{n} \xi_{i}^{2}$, for some constant $\alpha$ in the range $0<\alpha \leqq 1 / n$, and the normalization $\sum_{i=1}^{n} a_{i i}=1$. It is well known [1] - [4] that such differential operators enjoy the following strong minimum and boundary point principles: A nonconstant, twice differentiable function $u(x)$, satisfying $L u \leqq 0$ in $D$, cannot attain a local minimum in $D$. Moreover if $u$ attains a local minimum at a boundary point $x^{0}$ where $\partial D$ has the inner sphere property, and if $\nu$ is a unit vector directed internally to the sphere, then

$$
\liminf _{t \rightarrow 0^{+}}\left\{\frac{u\left(x^{0}+t v\right)-u\left(x^{0}\right)}{t}\right\}>0 .
$$

Equivalently, the boundary point principle states that for $\|x-x\|$ sufficiently small there exists a positive constant $m$ (depending upon v) such that

$$
u(x) \geqq u\left(x^{0}\right)+m\left\|x-x^{0}\right\|
$$

along the line $x^{0}+t \nu$. In this note we wish to obtain, for the case of a plane domain $(n=2)$, an analogous lower bound for the approach of $u(x)$ to a minimum occurring on the boundary when the inner sphere property is replaced by an inner cone (sector) property. The proof is based upon a comparison with a barrier function which has recently been obtained [5] for the class $\mathfrak{L}_{\alpha}$ in a plane sector with the aid of elliptic extremal operators [6]. Our result is the best possible for the class of differential operators $\mathfrak{L}_{\alpha}$ and moreover shows explicitly the dependence upon the ellipticity constant $\alpha$.

2. We shall first describe our barrier function for the plane sector

$$
S\left(\theta_{0}\right)=\left\{(x, y): r>0,|\theta|<\theta_{0}<\pi\right\}
$$

where $r, \theta$ denote the polar coordinates of the point $(x, y)$.

${ }^{1}$ Research supported in part by the Air Force Office of Scientific Research under grant AFOSR 1122-67. 


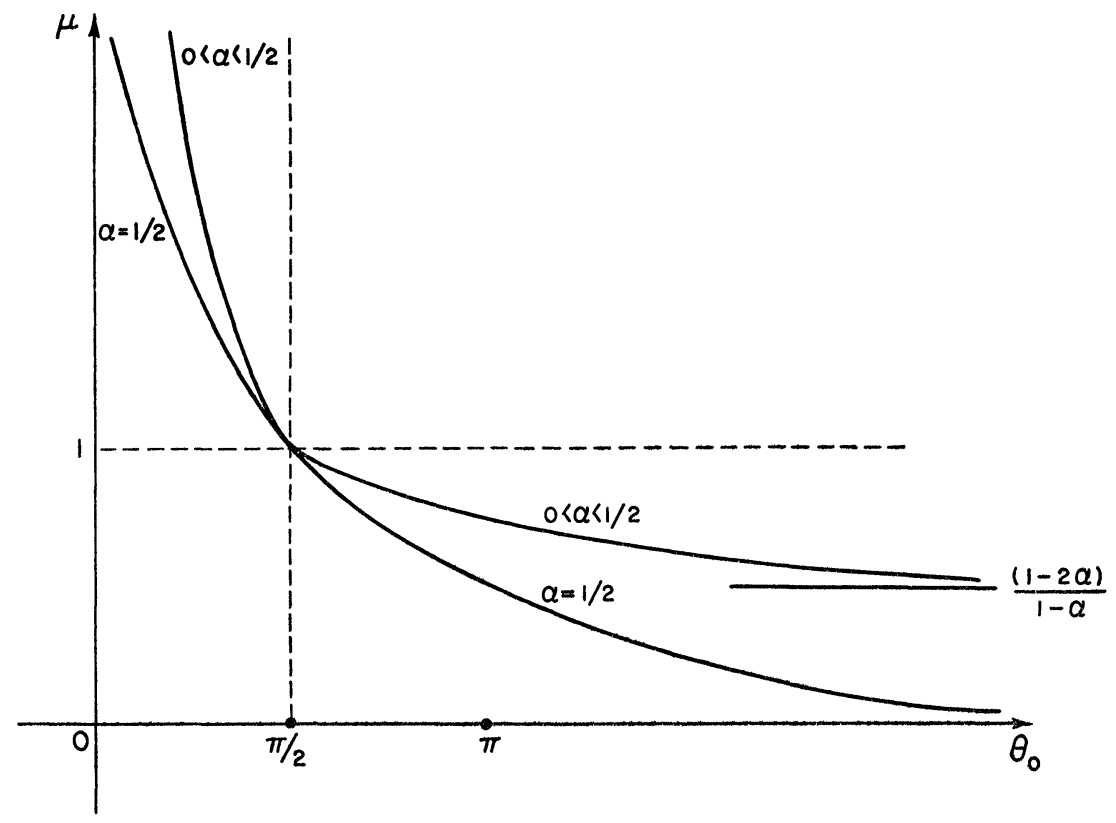

Figure 1

For the class of differential operators $\&_{\alpha}$ with $0<\alpha<\frac{1}{2}$ we define the constants $\zeta_{1}=\cos ^{-1}(1-2 \alpha) \in(0, \pi / 2), \zeta_{2}=\pi-\zeta_{1}$, and

$$
\mu=\frac{2(1-2 \alpha)}{\cos \zeta+(1-2 \alpha)}
$$

where:

(i) $\zeta \in\left(\zeta_{1}, \zeta_{2}\right)$ is the solution of $\zeta \tan \zeta_{1} / \tan \zeta+\zeta_{2}=2 \theta_{0}$ if $0<2 \theta_{0} \leqq \pi$; or

(ii) $\zeta \in\left(0, \zeta_{1}\right)$ is the solution of $(\pi-\zeta) \tan \zeta_{1} / \tan \zeta+\zeta_{1}=2 \theta_{0}$ if $\pi<2 \theta_{0}<2 \pi$.

For the class $\mathfrak{L}_{1 / 2}$, which consists of the single operator $\left\{\partial^{2} / \partial x^{2}+\partial^{2} / \partial y^{2}\right\} / 2$, we define $\mu=\pi / 2 \theta_{0}$.

A sketch of $\mu$ as a function of $\alpha$ and $\theta_{0}$ is shown in Figure 1. Note that $\mu$ is a monotone decreasing function of $\theta_{0}$ and $\mu(\alpha, \pi / 2)=1$.

Next we define the periodic function $C\left(\theta ; \theta_{0} ; \alpha\right)$ in the parametric form

$$
\begin{aligned}
C\left(\theta ; \theta_{0} ; \alpha\right) & =\frac{\cos \varphi\left\{1-\nu_{1} \cos \varphi\right\}^{(|\mu-1|-1) / 2}}{\left\{1-\nu_{2} \cos \varphi\right\}^{(|\mu-1|+1) / 2}} \\
\theta & =\frac{(4 \alpha(1-\alpha))^{1 / 2}}{\mu} \int_{0}^{\varphi} \frac{d \xi}{\left(1-\nu_{1} \cos \xi\right)\left(1-\nu_{2} \cos \xi\right)}
\end{aligned}
$$


where

$$
\nu_{1}=\frac{(|\mu-1|-1)(1-2 \alpha)}{\mu} \text { and } \nu_{2}=\frac{(|\mu-1|+1)(1-2 \alpha)}{\mu} .
$$

The following properties of $C\left(\theta ; \theta_{0} ; \alpha\right)$ are easily established:

(a) $C\left(\theta ; \theta_{0} ; 1 / 2\right)=\cos \mu \theta=\cos \left(\pi \theta / 2 \theta_{0}\right)$;

(b) $C(\theta ; \pi / 2 ; \alpha)=(\cos \theta) /(4 \alpha(1-\alpha))^{1 / 2}$;

(c) $C\left(\theta ; \theta_{0} ; \alpha\right)>0$ for $|\theta|<\theta_{0}$ and $C\left( \pm \theta_{0} ; \theta_{0} ; \alpha\right)=0$;

(d) $C\left(\theta ; \theta_{0} ; \alpha\right) \sim \cos \left(\pi \theta / 2 \theta_{0}\right) \quad$ as $\theta \rightarrow \pm \theta_{0}$.

The function

$$
v(x, y)=r^{\mu} C\left(\theta ; \theta_{0} ; \alpha\right),
$$

positive in the sector $S\left(\theta_{0}\right)$ and vanishing on its sides, is the barrier which we seek. It has been obtained in [5] as a solution of the minimizing equation relative to the class $\mathfrak{L}_{\alpha}$. It follows from the theory of extremal operators [6] that for every operator $L \in \mathfrak{L}_{\alpha}$ we have

$$
L v(x, y) \geqq 0 \quad \forall(x, y) \in S\left(\theta_{0}\right) .
$$

Furthermore there exists an operator $L^{\prime} \in \mathscr{L}_{\alpha}$ such that $L^{\prime} v=0$ in $S\left(\theta_{0}\right)$.

3. We now state our main result.

THEOREM. Let $D$ be an open subset of the plane and let $u(x, y)$ be a nonconstant, twice differentiable function in $D$ which is continuous on $\bar{D}$ and satisfies $L u \leqq 0$ in $D$ for some $L \in \mathfrak{L}_{\alpha}$. Suppose that $u$ attains a local minimum of $u_{0}$ at a boundary point $P_{0}$ which subtends an open truncated sector $S, S \subset D$, of half angle $\theta_{0}$. Then there exists a neighborhood $\Omega$ of $P_{0}$ and a positive constant $m$ such that

$$
u(x, y) \geqq u_{0}+m r^{\mu} C\left(\theta ; \theta_{0} ; \alpha\right) \text { in } \bar{S} \cap \bar{\Omega},
$$

where $r, \theta$ are polar coordinates measured from the vertex and axis of $S$ and $\mu, C$ are defined by (2.1) and (2.2) respectively.

Proof. Since the class $\mathscr{L}_{\alpha}$ is invariant under translation or rotation of coordinates there is no loss of generality in assuming $P_{0}$ to be the origin and the axis of $S$ to be the $x$-axis so that $r, \theta$ become the usual polar coordinates.

By the hypotheses there exists an $R>0$ such that $u \geqq u_{0}$ in $\left[S\left(\theta_{0}\right)\right]^{-}$ $\cap\{r \leqq R\}$. Since $u(x, y)$ is not identically constant we conclude from the strong minimum principle, the boundary point principle, and the property $(2.3)(\mathrm{d})$ that 


$$
m=R^{-\mu} \inf _{s(\theta 0) \cap\{r=R\}}\left\{\frac{u(x, y)-u_{0}}{C\left(\theta ; \theta_{0} ; \alpha\right)}\right\}
$$

is a positive constant. Let us define

$$
w(x, y)=u(x, y)-u_{0}-m v(x, y)
$$

in $\left[S\left(\theta_{0}\right)\right]-\cap\{r \leqq R\}$, where $v$ is our barrier given by (2.4).

In $S\left(\theta_{0}\right) \cap\{r<R\}$ we have $L w=L u-m L v \leqq 0$ while $w=\left(u-u_{0}\right) \geqq 0$ on $\partial S\left(\theta_{0}\right) \cap\{r \leqq R\}$ and, by (3.2), $w \geqq 0$ on $S\left(\theta_{0}\right) \cap\{r=R\}$. It follows from the minimum principle that $w \geqq 0$ in $\left[S\left(\theta_{0}\right)\right]-\cap\{r \leqq R\}$, which is the desired result.

REMARKs. (1) Since the barrier function $v=r^{\mu} C\left(\theta ; \theta_{0} ; \alpha\right)$ is itself a solution of $L^{\prime} u=0$ in $S\left(\theta_{0}\right)$ for some $L^{\prime} \in \mathscr{L}_{\alpha}$ our result cannot be improved.

(2) Note that $\mu=1$ for $\theta_{0}=\pi / 2, \mu>1$ for $\theta_{0}<\pi / 2$, and $\mu<1$ for $\theta_{0}>\pi / 2$. Thus for $\theta_{0}=\pi / 2$ our result coincides with that of the boundary point principle. When $\theta_{0}<\pi / 2$ the difference quotient $\left(u-u_{0}\right) / r$ may tend to zero when $r \rightarrow 0$, as is well known for domains without the inner sphere property. Note however that for $\theta_{0}>\pi / 2$ this difference quotient is unbounded as $r \rightarrow 0$ so that the theorem implies that no interior directional derivative can exist at a local minimum occurring at the vertex of an obtuse angle.

4. Suppose now that the hypotheses of the theorem hold for an operator $L^{*}$ of the form

$$
L^{*}=L+b_{1} \partial / \partial x+b_{2} \partial / \partial y
$$

with $L \in \mathscr{L}_{\alpha}$ and $\left(b_{1}^{2}+b_{2}^{2}\right)^{1 / 2}=o\{1 / r\}$ as $r \rightarrow 0$, where $r$ denotes distance measured from the boundary point $P_{0}$.

Let a fixed $\epsilon>0$ be given and denote by $\Omega^{*}$ the corresponding neighborhood of $P_{0}$ in which $\left(b_{1}^{2}+b_{2}^{2}\right)^{1 / 2} \leqq \epsilon / r$. Using again the minimizing operator relative to the class $\mathfrak{L}_{\alpha}$ it may be shown that there exists a function $T_{\epsilon}(\theta)$, with properties similar to those of $(2.3)$ (c) and (d), such that $v_{\epsilon}=r^{\mu+\epsilon} T_{\epsilon}(\theta)$ is a barrier for $L^{*}$ in $S \cap \Omega^{*}$. It follows that our theorem may be extended to the operator $L^{*}$, with the conclusion (3.1) replaced by

$$
u(x, y) \geqq u_{0}+m r^{\mu+e} T_{\epsilon}(\theta) \quad \text { in } \bar{S} \cap \bar{\Omega} .
$$

The results (3.1) or (4.1) are also valid for the operators $L+c$ or $L^{*}+c$, respectively, if $c \leqq 0$ in a neighborhood of $P_{0}$, provided that we assume that the minimum value $u_{0}$ is negative. $\left(u_{0} \leqq 0\right.$ is sufficient if in addition the growth of $|c|$ is suitably limited near $P_{0}$; e.g., if $c$ is bounded below.) 
5. Our results may also be applied to certain quasilinear equations. As an example let us consider a nonconstant solution $\phi(x, y)$ of the equation of minimal surfaces

$$
\left(1+\phi_{y}^{2}\right) \phi_{x x}-2 \phi_{x} \phi_{y} \phi_{x y}+\left(1+\phi_{x}^{2}\right) \phi_{y y}=0
$$

in the sector $S\left(\theta_{0}\right)$. If the gradient of $\phi$ is bounded

$$
|\operatorname{grad} \phi| \leqq M
$$

then $\phi$ satisfies the linear equation $L \phi=0$, where

$$
\begin{aligned}
L=\{ & \left(1+\phi_{y}^{2}\right) \partial^{2} / \partial x^{2}-2 \phi_{x} \phi_{y} \partial^{2} / \partial x \partial y \\
& \left.+\left(1+\phi_{x}^{2}\right) \partial^{2} / \partial y^{2}\right\} /\left\{2+|\operatorname{grad} \phi|^{2}\right\}
\end{aligned}
$$

is in the class $\mathfrak{L}_{\alpha}$ with

$$
\alpha=1 /\left(M^{2}+2\right) \text {. }
$$

If $\phi$ achieves a local minimum of $\phi_{0}$ at the origin then in a neighborhood of the origin we have, from (3.1) and (5.1),

$$
\phi_{0}+m r^{\mu} C\left(\theta ; \theta_{0} ; \alpha\right) \leqq \phi(x, y) \leqq \phi_{0}+M r, \quad m>0,
$$

where $\mu$ and $C$ are defined by (2.1) and (2.2). Note that if $\theta_{0}>\pi / 2$ then (5.3) yields a contradiction since $\mu<1$. As a result we may state: A nonconstant minimal surface with bounded gradient cannot attain a local minimum at the vertex of an obtuse angle.

\section{REFERENCES}

1. E. Hopf, Elementare Bermerkungen über die Lösungen partieller Differentialgleichungen zweiter Ordnung vom elliptischen Typus, S.-B. Preuss, Akad. Wiss. Berlin 19 (1927), 147-152.

2. - A remark on elliptic differential equations of second order, Proc. Amer. Math. Soc. 3 (1952), 791-793.

3. O. A. Oleinik, On properties of solutions of some boundary value problems for equations of elliptic type, Mat. Sb. 30 (1952), 695-712 (Russian).

4. C. Pucci, Proprieta di massimo e minimo delle soluzioni di eguazioni a derivate parziali del secondo ordine di tipo ellittico e parabolico, Rend. Accad. Naz. Lincei (8) 23 (1957); 24 (1958).

5. J. K. Oddson, Some solutions of elliptic extremal equations in the plane (to appear).

6. C. Pucci, Operatori ellittici estremanti, Ann. Mat. Pura Appl. 72 (1966), 141170.

University of California, Riverside 